\title{
Diagnosis of plant-wide oscillation through data-driven analysis and process understanding
}

\author{
Nina F. Thornhill ${ }^{\mathrm{a}, *}$, John W. Cox ${ }^{\mathrm{b}}$, Michael A. Paulonis ${ }^{\mathrm{b}}$ \\ ${ }^{a}$ Department of Electronic and Electrical Engineering, University College London, Torrington Place, London WC1E 7JE, UK \\ ${ }^{\mathrm{b}}$ Eastman Chemical Company, PO Box 431, Kingsport, TN 37662, USA
}

Received 11 November 2002; received in revised form 18 April 2003; accepted 21 April 2003

\begin{abstract}
Disturbances that propagate throughout a plant due to recycle streams, heat integration or other means can have an especially large impact on product quality and running costs. There is thus a motivation for automated detection of a plant-wide disturbance and for determination of the root cause so that the disturbance may be removed. In this article, data-driven techniques are used to diagnose a plant-wide oscillation in an Eastman Chemical Company plant. A numerical non-linearity index derived from routine measurements was able to suggest the root cause. Process understanding possessed by the plant control engineers then enhanced the data-driven analysis, for instance by identifying a proxy measurement for an unmeasured flow through the valve suspected of being the root cause. In situ tests of just one valve confirmed the suspected root cause and the plant-wide oscillation disappeared after repairing the valve. The diagnosis was right first time and the maintenance effort was thus minimized. The success of the study suggests there exists a fruitful direction for future research in the automated linkage of data-driven analysis with information about the structure and connectivity of the process.
\end{abstract}

(C) 2003 Elsevier Ltd. All rights reserved.

Keywords: Chemical industry; Condition monitoring; Control loop performance; Fault diagnosis; Non-linearity; Plant-wide disturbance; Power spectrum; Process control; Surrogate data

\section{Introduction}

It is important to detect and diagnose the causes of oscillations in a chemical process because a plant running close to product quality limits or operating constraints is more profitable than a plant that has to back away due to the amplitude of the oscillations (Martin, Turpin, \& Cline 1991; Shunta, 1995). As economic issues force plants to be more efficient, process features such as recycle streams and heat integration are increasingly utilized. These features complicate process control because variation can propagate through the plant in complex ways, often turning a single source of variation into a plant-wide oscillation. In these cases, a key issue is to determine the root cause (Qin, 1998; Paulonis \& Cox, 2003). Unfortunately, it is rarely easy to determine cause and effect when an oscillation is present throughout the plant.

*Corresponding author. Tel.: +44-20-7679-3983; fax: +44-20-73889325.

E-mail address: n.thornhill@ee.ucl.ac.uk (N.F. Thornhill).
Root cause diagnosis is enhanced by knowledge of the process, for example the connectivity and directions of flow of materials and heat. Such process understanding provides an explanation of the means by which an oscillation propagates from the root cause to other locations. This paper shows how data-driven methods in combination with process understanding led to efficient root cause diagnosis in an industrial case study at Eastman Chemical Company. The root cause of a plant-wide oscillatory disturbance was determined, verified and corrected and its means of propagation understood. The success of the study suggests there is a need for a linkage of the cause and effect information held in a process flowsheet or model with the results of analysis of operating data.

The steps taken in the study are outlined below. The first stage used data from routine process operation as follows:

- Detection of a plant-wide oscillation by means of automated grouping of oscillations with similar periods, and a determination of the severity of the oscillation. 
- Preliminary diagnosis of the root cause of the oscillation using a non-linearity index that distinguished between the root cause and secondary oscillations.

Analysis based upon routine operating data can yield information-rich statistics such as the non-linearity index which focus a process control engineer's attention on the part of the plant where the problem lies. The engineer can then integrate the findings with his or her understanding of the process to specify additional tests and order maintenance actions. In this case the additional insights achieved were:

- Identification of a proxy variable for an unmeasured flow through a control valve, which enabled the input-output characteristic of the valve to be examined.

- Verification that a mechanism existed for the oscillation from the suspected root cause to propagate plant-wide.

The diagnosis was finalized by means of a set of special tests and the faulty valve was then prioritized for maintenance.

Section 2 reviews data-driven techniques for characterization and diagnosis of oscillations. Section 3 introduces the industrial process, while Section 4 presents the non-linearity diagnosis procedure. Section 5 shows how a candidate for the root cause was identified and the further role played by knowledge and understanding of the process. Section 4 describes the maintenance action taken and illustrates the improvement in the plant operation after maintenance. The paper ends with conclusions and recommendations.

\section{Background}

\subsection{Oscillation detection}

Several authors have addressed the detection of oscillatory measurements in process data. Hägglund (1995) described a method for the detection of oscillations within a control loop and other authors have also considered the problem (Thornhill \& Hägglund, 1997; Forsman \& Stattin, 1999; Rengaswamy, Hagglund, \& Venkatasubramanian, 2001a). Miao and Seborg (1999) considered the absolute value of the oscillatory autocovariance function at the first minimum together with additional cycles. Xia and Howell (2003) gave an analysis of loop status that determined if an individual loop was affected by long- or short-term transients or a slow or fast oscillatory disturbance. These approaches were aimed at individual control loops.
When an oscillation has been detected in one control loop then it is necessary to find all the measurements or control loops having the same disturbance because the root cause will be among that group. Detection of plant-wide oscillation was considered by Thornhill, Huang, and Zhang (2003), who described a method for detection of groups of similar oscillations based upon the zero crossings of filtered autocovariance functions.

\subsection{Oscillation diagnosis}

It is beneficial to track down the root cause of a plantwide problem because then the maintenance effort can be effectively directed towards the equipment or control loop that needs it. A common source of oscillation is a limit cycle caused by a control valve with a deadband or excessive static friction (Åström, 1991; Shinskey, 2000). A process variable oscillating for that reason can readily propagate the oscillation to other variables and disturb other control loops, hence causing a plant-wide disturbance. A focus upon non-linear root causes can thus be justified because valve friction causes the majority of cases, according to reported surveys (Bialkowski, 1992; Ender, 1993).

Thornhill and Hägglund (1997), Ruel and Gerry (1998) and Thornhill, Shah, and Huang (2001) described the diagnosis of root causes by observation of the spectral harmonics of periodic limit cycles. Advanced methods based upon higher order spectra are also staring to be used for control loop diagnosis (Choudhury, Shah, \& Thornhill, 2002).

Maps of process variable versus manipulated variable (i.e. flow through the valve versus valve position) can be utilized to diagnose the nature of a valve fault, and are used in several commercial packages. Other signatures such as odd and even cross correlations and an assessment of the probability density function of the measurement values (Horch, 1999) have also been used. However, those techniques rely upon a measurement of flow through the valve that is not always present in an industrial plant.

The aim of this paper is to enable a precise and rapid diagnosis by means of plant-wide non-linearity testing.

The non-linearity test determines whether a time series could plausibly be the output of a linear system driven by Gaussian white noise, or whether its properties could only be explained as the output of a non-linear system (Theiler, Eubank, Longtin, \& Galdrikian, 1992; Kantz \& Schreiber, 1997; Schreiber \& Schmitz, 2000). The underlying concept is that non-linearity reduces due to the filtering nature of the process as the disturbance propagates away from the root cause. Therefore, the root cause is to be found in the part of the plant where the non-linearity of the time series of the plant 
measurements is highest. This statement will be justified in Section 4.1.

\section{The industrial process}

\subsection{The company}

The Advanced Controls Technology group of Eastman Chemical Company has an interest in monitoring the performance of control loops on Eastman plants around the world. This is made possible by an advanced in-house automated controller performance assessment system (Paulonis \& Cox, 2003). The system has incorporated Eastman's own approaches and some of the techniques highlighted in Section 2 and elsewhere in the process control literature. Benefits of the system were recently reported. For example, off-class production due to process-control-related causes was reduced by $53 \%$ in one plant.

The Advanced Controls Technology group has identified a need in the system for diagnosis by identifying sets of loops that appear to share a common disturbance and identification of a loop that may be the root cause of the distributed disturbance.

\subsection{Automatic control valves}

It is well known that friction in a valve can cause a sustained limit-cycle oscillation in control loops (Piipponen, 1996; McMillan, 1995). Friction becomes more significant as the valve condition deteriorates and its effect may also be exacerbated if the valve gland nut is tightened to compress the packing in order to reduce leaks and emissions.

A description of pneumatic control valves may be found in Whalen (1983). The primary actuation is air pressure exerting a force on a diaphragm which is attached the top of the valve stem. The principle of operation is that a valve plug attached to a stem moves relative to a fixed valve seat in order to restrict the flow of fluid through the valve. Generally, pushing down on the stem closes the valve while if no force is applied to the stem then the valve should open fully due to the action of a return spring. However, the stem is in contact with the valve packing material and thus there can be significant friction forces.

The forces balance when the valve is not moving:

$F_{a}=k x+F_{f}$,

where $x$ is the stem position, $F_{a}$ is the force applied by the pneumatic actuator, $k x$ is the spring force with $k$ being the spring constant and $F_{f}$ is the friction force. A typical friction-velocity characteristic is shown in Fig. 1 showing manufacturers' values reported by Kayihan and Doyle (2000). A key feature of the friction

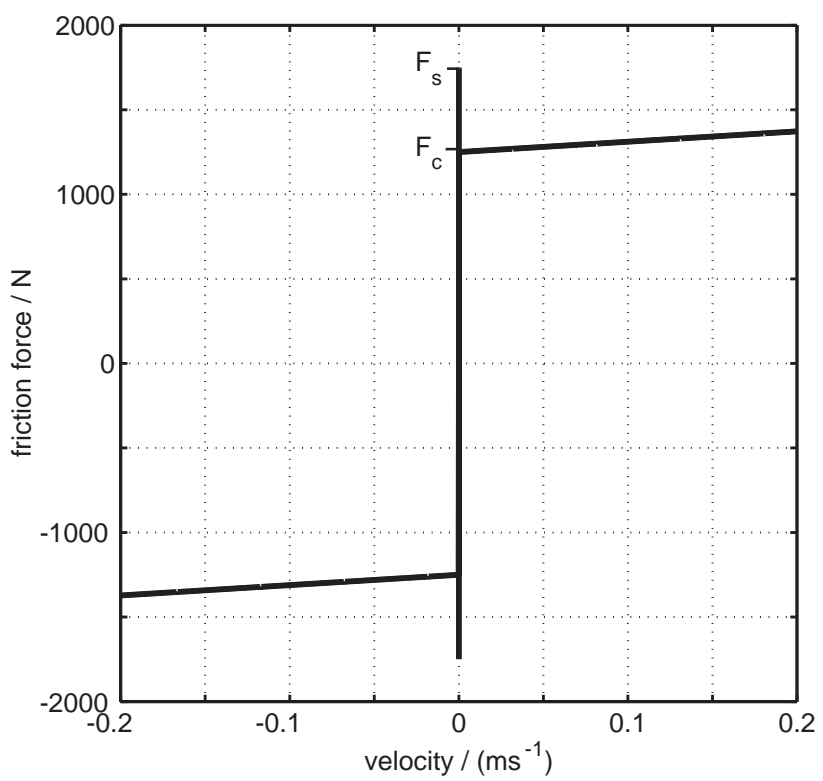

Fig. 1. A typical control valve friction versus velocity characteristic.

characteristic is that there is a range of values for the friction force between $\pm F_{s}$ when the velocity is zero. The valve thus has a deadband because there is no response from the valve until $\left|F_{a}-k x\right|>F_{s}$. There may also be stick-slip behavior if the initial moving friction $F_{c}$ is less than the maximum static friction $F_{s}$.

\subsection{The process}

Schematic: The schematic of the Eastman Chemical Company plant under study is shown in Fig. 2. The process features three distillation columns, two decanters and several recycle streams. There are 15 control loops and 15 indicators that are numbered from 1 to 30 on the schematic. Six of the eight flow controllers are in a cascade configuration, therefore their set points ( $s p$ 's) as well as the process variables ( $p v$ 's) and controller outputs (op's) are time varying.

Data set: Uncompressed plant data were sampled from the control system every $20 \mathrm{~s}$ for each of the indicators and for the set point, measurement, and output of the control loops. Fig. 3 shows the measurements ( $p v$ 's) from 2 days of running for all 30 plant tags. Fig. 3 also shows the controller outputs (op's) for the tags that are under automatic control. All time trends were scaled to unit standard deviation.

Visual inspection of the time trend plots in Fig. 3 shows the presence of oscillations with a period of nearly $2 \mathrm{~h}$ (340 sample per cycle). This oscillation affects many $p v$ 's and $o p$ 's and is therefore a plant-wide oscillation. It can be seen that the disturbance affects 


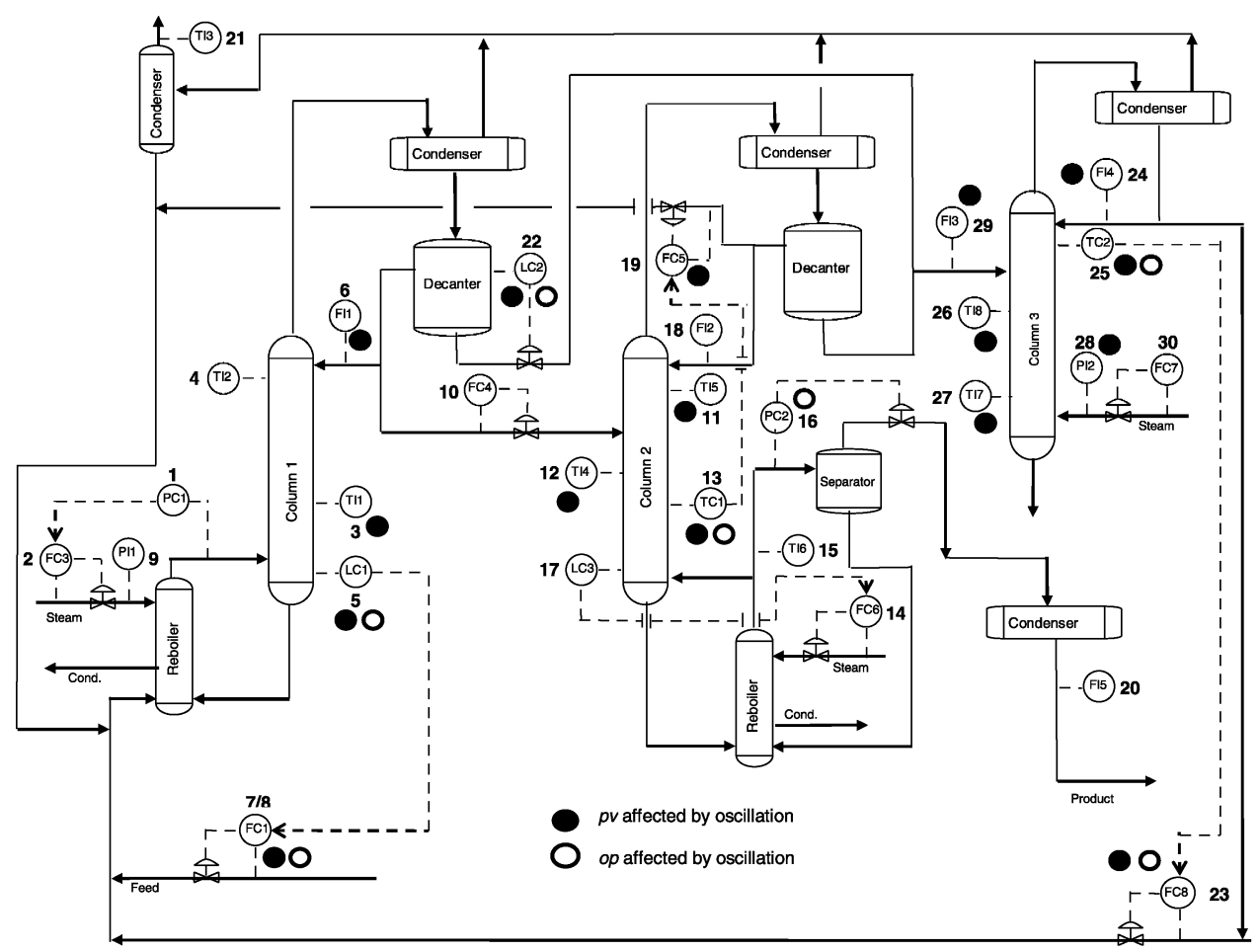

Fig. 2. Process schematic. The circular symbols show the tags affected by a plant-wide oscillation.

column temperature, thus causing variability in the product composition. The disturbance also affects column loading in a periodic way and limits production rate. Therefore, there is an economic incentive to determine the cause and remove it. The challenge is to determine the likely origin of the oscillation from routine operating data so that maintenance effort can be directed efficiently.

The detection of the oscillatory plant-wide disturbances in Fig. 3 was reported by Thornhill et al. (2003), who inspected the regularity of the zero crossings of autocovariance functions of the measurements of the process variables $(p v)$ and provided an automated means of grouping oscillations with similar periods. The method also determined the percentage spectral power associated with the oscillation: for example, $100 \%$ power in an oscillation would mean there were no other oscillations present in the measurement and no noise.

\section{Non-linearity diagnosis}

\subsection{Non-linearity in process time trends}

A linear time series has a linear dynamic model such as the Box Jenkins model with constant coefficients driven by Gaussian white noise. By contrast, the non-linear time series of interest in this work have a non-linear feedback function:

$$
\begin{aligned}
& x(n)=\phi(x(n-1), x(n-2), \ldots, u(n-1))+w(n), \\
& y(n)=h(x(n))+v(n), \\
& u(n)=g(y(n)),
\end{aligned}
$$

where $\phi(x(n-1), x(n-2), \ldots, u(n-1))$ represents linear dynamics, $x(n)$ is an internal state, $y(n)$ is a measurement from the process, $h(x(n))$ is a measurement function that may be linear or non-linear, $g(x(n))$ is a non-linear feedback function and $w(n)$ and $v(n)$ are process and measurement noises. An example of a non-linear feedback function is the on-off control of a directly injected steam heated tank in which the steam valve switches on when the temperature drops to a low limit and switches off again when the temperature reaches a high limit. The non-linear characteristic $g$ in that case is a relay with deadband. The temperature is not steady in such a system and it cycles in periodic pattern. Control loops having valves with non-linear friction characteristics and instrumentation faults can lead to similar behavior and are described by the same model structure.

The basis of the diagnostic method is an index for non-linearity that grows stronger closer to the source. Suppose, for instance, that the limit cycle originates in a flow loop and has a square-ish waveform such as is common in a proportional plus integral flow control loop that has a valve with excessive static friction. A square wave has harmonics at multiples of the 
normalised $\mathrm{pv}$

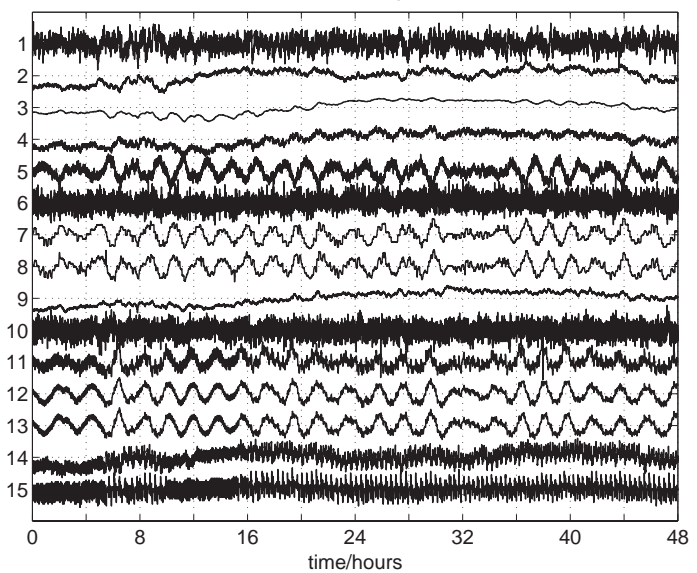

normalised op

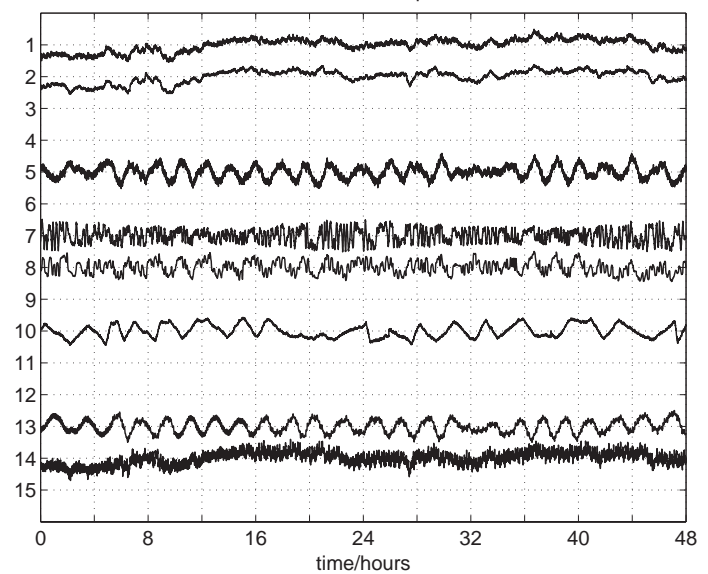

normalised $\mathrm{pv}$

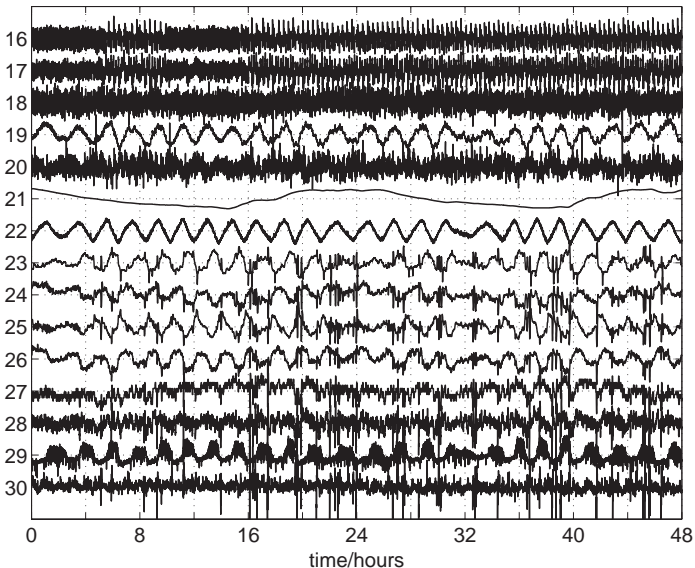

normalised op

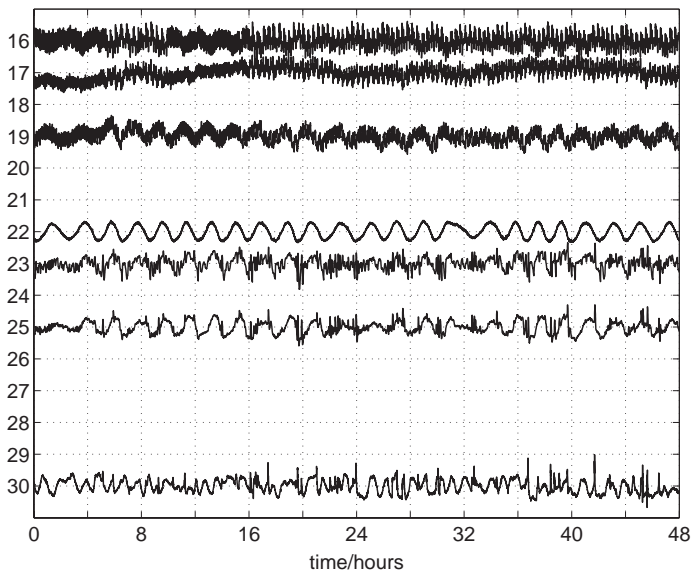

Fig. 3. Normalized time trends of $p v$ and $o p$ of 15 controllers and $p v$ of 15 plant instruments.

fundamental oscillation frequency, and moreover the phases of the harmonics are coupled so that the phase of each harmonic is determined by the phases of others. The non-linear root cause may be inferred from the presence of harmonics and phase coupling in the flow measurement.

The reason why the non-linearity is strongest nearest to the source is that the plant acts as a mechanical lowpass filter. As the disturbance influences other variables such as levels, compositions and temperatures the waveforms become more sinusoidal and more linear because low-pass plant dynamics removes the higher harmonics and destroys the phase coupling.

The non-linearity test determines whether a time series could plausibly be the output of a linear system driven by Gaussian white noise, or whether its properties can only be explained as the output of non-linearity.

\subsection{Non-linearity test}

The test statistic used is the root mean square (r.m.s.) value of the error from non-linear prediction using matching of nearest neighbors in an $m$-dimensional phase space known as an embedding (for instance, a plot of $y(n)$ versus $y(n-d)$ for some delay $d$ would be a twodimensional embedding).

Fig. 4 gives a visual illustration of the concept. The top panel is an oscillatory time trend from a refinery (courtesy of BP Oil). It has a clearly defined pattern. Therefore, a good prediction of where the trend will go after reaching a given position, for example at one of ringed peaks, can be achieved by locating similar peaks in the time trend and observing where the trend went next on those occasions.

The lower left panel in Fig. 4 labeled "test data" shows an embedded plot in which the trend is plotted against a delayed version of itself. In that case the embedded data matrix was

$$
Y=\left(\begin{array}{cc}
y(1) & y(4) \\
y(2) & y(5) \\
y(3) & y(6) \\
\vdots & \vdots \\
y(N-3) & y(N)
\end{array}\right) .
$$




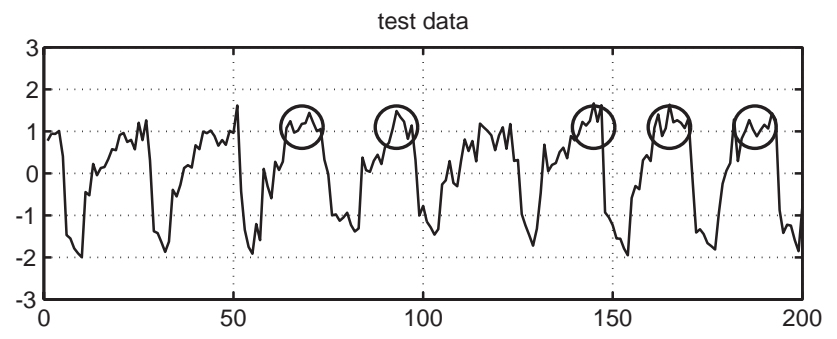

typical surrogate
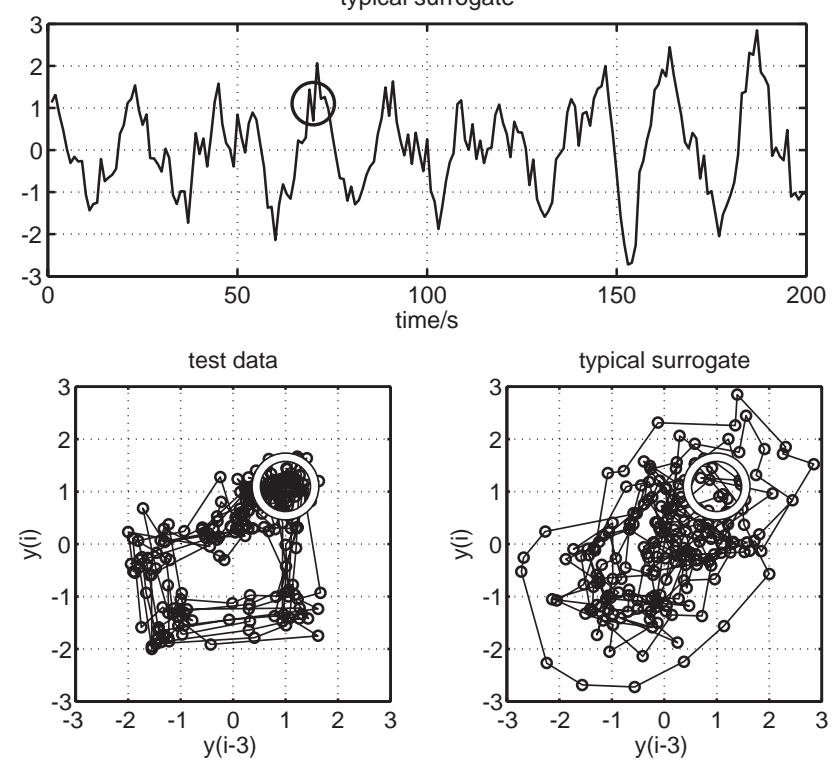

Fig. 4. A limit-cycle oscillation and a typical surrogate represented as time trends (above) and as a two-dimensional embedding (below). The white rings in the embedded plots indicate the positions of the peaks circled in the time trends.

Each plotted point represents one row of the data matrix and the trajectory is parameterized by time. The peaks of the time trend are in the region of the embedded plot highlighted with a white ring. The prediction task involves identifying trajectories through the highlighted region that are similar to the current trajectory because the paths those trajectories took after they left the region provide a good prediction of where the current trajectory will go next. The predictions are reliable because the plot has structure, for instance there are regions of the space that are never visited.

The panels of Fig. 4 labeled "surrogate data" will be explained in Section 4.4.

\subsection{Optimization of parameters}

The non-linearity test is based upon Kantz and Schreiber (1997).

The embedded data matrix $Y$ has $E$ columns, each of which is a copy of the original data set delayed by one sampling interval. Each row of $Y$ thus represents a segment from the original time trend and is time shifted from the next row (segment) by one sampling interval.
Since the original data formed a continuous time trend the first and second rows are similar. They are called near-in-time neighbors. Also, if the time trend is oscillatory then later rows of $Y$ will be similar to the earlier rows after one or more complete cycles of oscillation. Those rows are called near neighbors.

$H$-step ahead predictions are generated from near neighbors with near-in-time neighbors excluded. For instance, if the first row were identified as a near neighbor of row 51 and if $H$ were 3 then $y(4)$ would give a prediction of $y(54)$. A more refined prediction for $y(54)$ would be the average of the predictions from $k$ nearest neighbors of row 51. A prediction error for every measured $y$-value can be created by subtracting its average prediction from its observed value and the overall prediction error for the data set is the r.m.s. value of all the prediction errors. The analysis is acausal and any element in the time series may be predicted from both earlier and later values.

The periodic nature of the oscillation has been exploited in order to give robust default settings for the parameters. The following pre-processing is recommended.

1. The number of samples per cycle, $S$, is adjusted to 25-35. The data set is subsampled if necessary. For instance, if the oscillation has 340 samples per cycle, then taking every tenth spot value gives a data subset with 34 samples per cycle.

2. The number of cycles of oscillation should be at least 10 .

3. The selected data are end-matched. It is necessary to find a subset of the data with a number of full cycles such that the starting gradient and value match well to the final gradient and value. This step is achieved using an automated search for a subset of the data $\left\{y_{i}, \ldots, y_{j}\right\}$ that minimizes $J_{i, j}$ below, where $\bar{y}_{i, j}$ is the mean value of the data subset. This step can be achieved by a program loop that runs through all candidate data subsets.

$$
J_{i, j}=\frac{\left(y_{i}-y_{j}\right)^{2}+\left(\left(y_{i+1}-y_{i}\right)-\left(y_{j}-y_{j-1}\right)\right)^{2}}{\sum_{k=i}^{j}\left(y_{k}-\bar{y}_{i, j}\right)^{2}} .
$$

4. The end-matched data are mean centered and scaled to unit standard deviation.

All of these pre-processing steps are readily automated for use by non-experts. It would, however, always be good practice to inspect plots of the pre-processed data to ensure that no set point changes or major disturbances were present.

Consistent and robust results have been achieved using $E=S$ and $H=E$. Thus, each row of the data matrix is one complete cycle of oscillation. The performance of the algorithm deteriorates if too large a value is used for the number of nearest neighbors, $k$. 
Table 1

Data-driven analysis of plant-wide oscillation with average period of 340 samples per cycle showing period, percentage power in the oscillation and non-linearity

\begin{tabular}{|c|c|c|c|c|c|c|c|c|c|c|c|c|c|c|}
\hline Tag & No. & Period & $\begin{array}{l}\text { Power } \\
(\%)\end{array}$ & $N$ & Tag & No. & Period & $\begin{array}{l}\text { Power } \\
(\%)\end{array}$ & $N$ & Tag & No. & Period & $\begin{array}{l}\text { Power } \\
(\%)\end{array}$ & $N$ \\
\hline TI1.PV & 3 & $326 \pm 10$ & 5 & - & TC1.OP & 13 & $372 \pm 51$ & 86 & - & FC8.OP & 23 & $336 \pm 14$ & 51 & 1.2 \\
\hline LC1.PV & 5 & $320 \pm 24$ & 82 & - & PC2.PV & 16 & - & - & - & FC8.SP & 23 & $347 \pm 30$ & 80 & 1.1 \\
\hline LC1.OP & 5 & $319 \pm 31$ & 86 & - & PC2.OP & 16 & $373 \pm 72$ & 17 & - & FI4.PV & 24 & $361 \pm 66$ & 43 & - \\
\hline FC1.PV & $7 / 8$ & $319 \pm 31$ & 87 & - & FC5.PV & 19 & $372 \pm 51$ & 87 & - & TC2.PV & 25 & $342 \pm 21$ & 71 & 1.0 \\
\hline FC1.OP & $7 / 8$ & $324 \pm 28$ & 64 & - & FC5.SP & 19 & $372 \pm 51$ & 86 & - & TC2.OP & 25 & $347 \pm 29$ & 80 & 1.1 \\
\hline FC1.SP & $7 / 8$ & $318 \pm 31$ & 89 & - & FI5.PV & 20 & $312 \pm 102$ & 15 & - & TI8.PV & 26 & $362 \pm 54$ & 76 & - \\
\hline TI5.PV & 11 & $315 \pm 53$ & 56 & - & LC2.PV & 22 & $362 \pm 36$ & 97 & 1.1 & TI7.PV & 27 & $322 \pm 22$ & 29 & - \\
\hline TI4.PV & 12 & $374 \pm 69$ & 90 & - & LC2.OP & 22 & $359 \pm 20$ & 98 & 1.3 & PI2.PV & 28 & $324 \pm 26$ & 22 & - \\
\hline TC1.PV & 13 & $373 \pm 68$ & 90 & - & FC8.PV & 23 & $347 \pm 31$ & 80 & 1.1 & FI3.PV & 29 & $369 \pm 72$ & 67 & 1.1 \\
\hline
\end{tabular}

A cautious and robust value of $k$ is 8 . Use of eight nearest neighbors covers the case when only nine cycles (with eight cycles to act as near neighbors) remain from the original 10 after the end-matching step.

\subsection{Statistical procedure}

Surrogate data are derived from the pre-processed time trend. Surrogate data have the same power spectrum as the time trend under test. The magnitudes of the discrete Fourier transform (DFT) are the same in both cases but the arguments of the DFT of the surrogate data set are randomized in order to destroy phase coupling.

A non-linear time series is more predictable than its surrogates. Fig. 4 shows the time trend and the embedded plot of surrogate data derived from the refinery test data. The surrogate is also oscillatory but its embedded plot (lower right-hand panel) lacks structure. In particular, the trajectories traverse through the white ring in the embedded plot along many different paths and leave in many different directions, thus the surrogate is less predictable than the test data.

The non-linearity test requires the determination of the r.m.s. prediction errors of $n$ surrogates. The statistical distribution of those errors gives a reference distribution. If the r.m.s. prediction error of the test data lies on the lower tail of the reference distribution then the test signal is more predictable than the surrogates and non-linearity is inferred. The non-linearity index is the following threesigma statistic:

$N=\frac{\bar{\Gamma}_{\text {surr }}-\Gamma_{\text {test }}}{3 \sigma_{\Gamma_{\text {surr }}}}$,

where $\Gamma_{\text {test }}$ is the r.m.s. prediction error of the test data, $\bar{\Gamma}_{\text {surr }}$ is the mean of the reference distribution and $\sigma_{\Gamma_{\text {surr }}}$ its standard deviation. If $N>1$ then non-linearity is inferred in the test time series.

The test is more stringent and discriminating than the one applied in an earlier report where a two-sigma test was used (Thornhill, Xia, Howell, Cox, \& Paulonis, 2002) because here a three-sigma test is in use. In addition, the procedure has now been optimized for use with limit cycles by exploitation of the oscillatory nature of the time trend.

\section{Diagnosis of plant-wide oscillation}

\subsection{Detection and characterization of oscillations}

The first step itemized in Section 1 was oscillation detection. Automated oscillation analysis confirmed the presence of the oscillations that can be seen in Fig. 3 and evaluated the power associated with them. A tag was judged to be participating in the plant-wide oscillation if it had more that $5 \%$ of its spectral power associated with the oscillation. The period was about 340 samples per cycle or $113 \mathrm{~min}$.

Table 1 gives the oscillation period in $p v$ and $o p$, and shows the percentage power associated with the oscillation, which was close to $100 \%$ in some cases. The high power indicates that finding the root cause of the oscillation would address much of the variability present in this plant. The percentage power associated both with $p v$ and $o p$ was highest for LC2 (Tag 22).

Fig. 2 shows the distribution of the plant-wide oscillation on the process schematic. The schematic indicates that the oscillation was widespread and also highlights instances of disturbance rejection. For example, the oscillation was removed effectively by controller PC2 (Tag 16) because its $p v$ was not oscillating but the $o p$ was oscillating.

It is noted that there were also other oscillations present. For instance, a faster oscillation was also found with a period of 64 samples per cycle $(21 \mathrm{~min})$ in $\mathrm{PC} 1$ (Tag 1), TI6 (Tag 15), LC3 (Tag 17) and PI2 (Tag 28). The focus here, however, is on the slower plant-wide oscillation with a period of $113 \mathrm{~min}$ because it is a prominent and widespread disturbance that accounts for most of the process variability. 


\subsection{Non-linearity testing}

Non-linearity testing was applied to give a preliminary diagnosis of the root cause. The non-linearity was determined from samples $720(4 \mathrm{~h})$ to $6000(33.3 \mathrm{~h})$ where the oscillations were well established. Table 1 gives the non-linearity indexes for cases where nonlinearity was detected. A dash means no non-linearity was detected. The uncertainty in the index is about \pm 0.1 .

The non-linearity was present in tags associated with column 3 and with the $p v$ and $o p$ of LC2. The non-linearity was highest in the op of LC2 and thus the control valve of LC2 is a candidate for the root cause.

\subsection{Application of process understanding}

The bad actor: The above data-driven analysis of routine operating data yielded rich information and focuses the process control engineers' attention on the part of the plant where the problem lies. The datadriven evidence points to LC2 (Tag 22) as the root cause. However, the high cost of plant tests and maintenance calls for a cautious "belt and braces" approach and further confirmation is needed before the costs of down-time and maintenance can be justified. At this stage it is useful to call upon the control engineers' understanding of the plant, especially the information they have about the connectivity of the plant.

The time trends associated with the control valve for LC2 were investigated and the need for a proxy for the unmeasured flow through the LC2 valve was identified. Examination of the flowsheet shows that FI3 (Tag 29) was equal to that flow plus material from the decanter for column 2. Therefore, FI3 was used to represent the flow through the control valve for LC2.

Fig. 5 shows the valve input (the $o p$ from LC2) versus the flow through the valve (FI3) and also plots their time trends. The valve has the signature of a deadband because although FI3 is noisy, it is clear that it tends to stay at a constant value whenever the valve input changes direction.

A valve with a deadband can cause persistent limitcycle oscillation, as discussed in Section 3.2. These results thus indicate that the cause of the plant-wide oscillation with a period of $113 \mathrm{~min}$ was the valve in the LC2 level control loop.

Mechanisms of propagation: The root cause diagnosis procedure is not complete until a feasible mechanism of propagation to all the tags suffering from secondary oscillations is explained. The process schematic shows that a mechanism exists for the disturbance from LC2 to
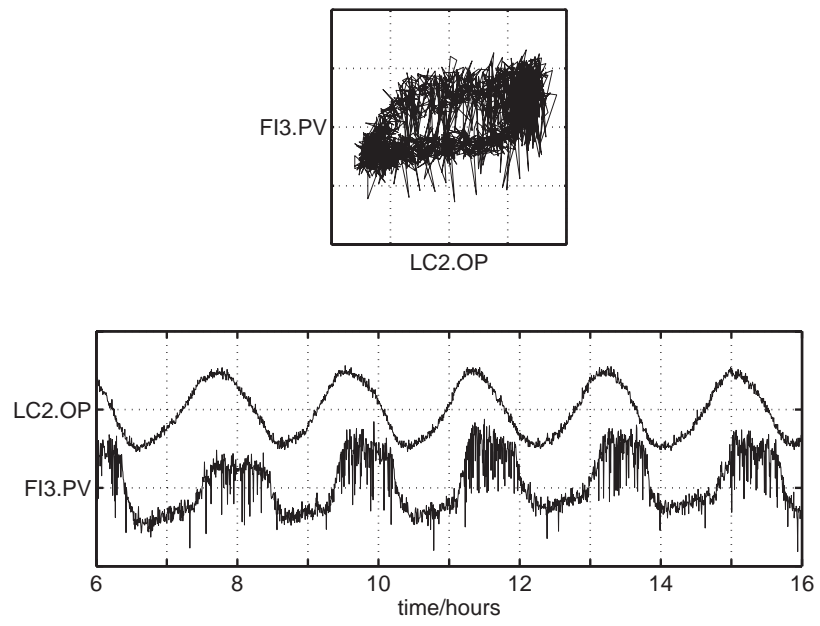

Fig. 5. Diagnosis of valve deadband using a proxy flow variable.

propagate to all the other tags, as follows:

- Uneven flow through the control valve of LC2 would affect FI3 (Tag 29) and propagate to column 3 including TC2 (Tag 25).

- Disturbance to TC2 would propagate to the cascade controller FC8 (Tag 23) and upset the recycle flow to column 1, and hence disturb the level controller LC1 (Tag 5).

- LC1 would adjust FC1 (Tags 7 and 8) to compensate for the disturbance to the recycle flow. It can be seen from Fig. 1 that FC1 (Tags 7 and 8) and FC8 (Tag 23) are almost in anti-phase.

- It is less obvious how uneven flow through the control valve of LC2 would upset column 2 since the feed flow (FC4, Tag 10 ) was not affected by the plant-wide oscillation and neither was the reflux flow (FI2, Tag 18). A likely mechanism is that the feed or reflux composition vary because of disturbance to the interface level in one or both decanters.

- Disturbance to FC5 (Tag 19) would also propagate to column 1 through the recycle, as described above for the FC8 recycle stream.

Other hypotheses can be ruled out because no mechanism exists for their propagation. For instance the data-driven results suggest the cascade control involving TC2 and FC8 should be considered because the $p v$ 's and $o p$ 's are non-linear although the percentage power is lower than for $\mathrm{LC} 2$.

Temperature control loop TC2 (Tag 25) could not be the root cause, however. TC2 influences recycle from column 3 to column 1 through the action of FC8, and could disturb TI7 and TI8 in column 3. However, it could not disturb FI3 because the feed to the column is determined only by conditions upstream. Likewise no mechanism exists for TC1 to influence LC2. The uneven flow of FC5 (Tag 19) might disturb the flow from the 
bottom of its decanter but it could not upset the level LC2 because flow from LC2's decanter is controlled.

\subsection{Confirmation of the root cause}

The evidence for the LC2 (Tag 22) control valve as the root cause was strong and justified further testing of the LC2 control valve. The plant was not near a planned shutdown so on-line tests were performed. The controller was put in manual and the valve was stepped through several travel cycles. A deadband of $4 \%$ was detected. Fig. 6 shows that when LC2 was put in manual and held constant at $2 \mathrm{~h}$ into the test the oscillation with a period of $113 \mathrm{~min}$ ( 340 samples per cycle) disappeared. It was put back in service at $4 \mathrm{~h}$ into the test with the controller gain doubled, and it was observed that the both the period and the magnitude of the oscillation decreased. These finding are consistent with the hypothesis of a deadband in the LC2 control valve. With a larger controller gain, the actuator force $F_{a}$ increases faster and traverses the valve deadband more rapidly. The flow through the control valve thus responds sooner when the level is not at its set point and thus there is a less severe deviation in the level of liquid in the LC2 decanter.

The above test confirmed the LC2 control valve was the origin of the limit cycle. During the period in manual (2-4h in Fig. 5) another oscillation with a different period could be observed in FI3 (Tag 29). Its period is about $20 \mathrm{~min}$ and it is therefore likely to be a secondary effect of the 64 sample per cycle oscillation mentioned in Section 3.3. Under closed loop operation, however, that oscillation cannot be seen in these tags.

The valve was scheduled for maintenance at the next shutdown. Fig. 7 shows the performance of key variables after the shutdown. It is clear the oscillation at 340 samples per cycle has been cured by the maintenance of control valve LC2. The vertical scales in Fig. 7 are matched for direct comparison such that, for instance, the vertical scale for Tag 22 is the same in the beforeand after-maintenance plots.

It is interesting to reflect that the solution of the plantwide oscillation could have been very costly without the guidance of the non-linearity test and the insight from the flowsheet that FI3 (Tag 19) was a proxy for the
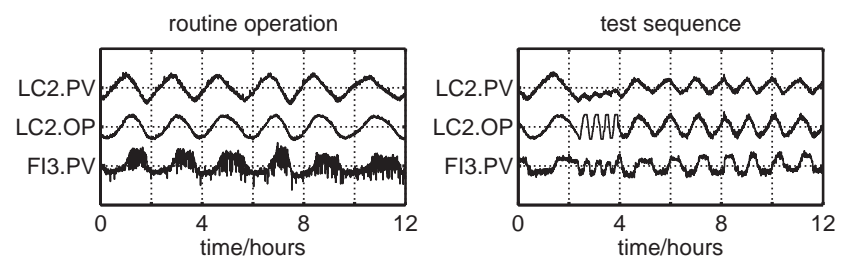

Fig. 6. Testing of the LC2 (Tag 22) control valve. The oscillation disappeared when the controller was placed in manual $2 \mathrm{~h}$ into the test sequence (right-hand plot). At $4 \mathrm{~h}$ the controller was put back in service with its gain doubled.
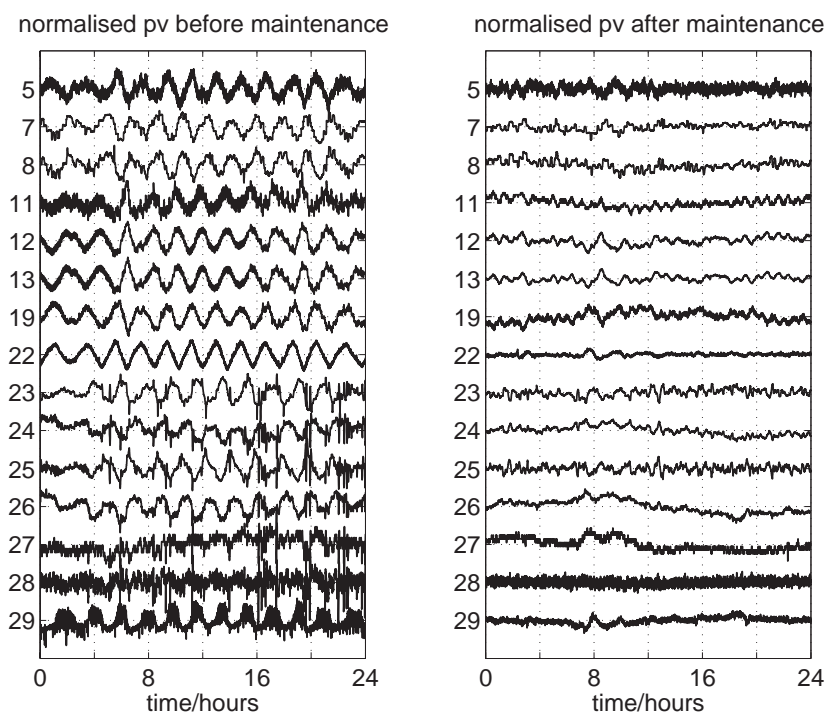

Fig. 7. Comparison of oscillating variables in the plant in the periods before and after the maintenance of control valve of LC2 (Tag 22). For each tag, the vertical axis scaling is the same in the before and after plots.

unmeasured flow. All the control valves participating in the plant-wide oscillation would have to be tested one by one until the root cause was found.

\section{Conclusion and comment}

This paper has shown how industrial process data, a toolkit of data analysis techniques, and process understanding were utilized to detect a disturbance that propagated plant-wide and to identify the root cause. The root cause of a disturbance affecting many of the controllers and indicators in the process was diagnosed correctly on the first attempt. At present, human interaction is required to aggregate and interpret the analysis results using process understanding to confirm the root cause diagnosis. The benefit of the present work is that the human interaction is with a small number of information-packed statistics. The methodology provides a foundation for future refinement such that a human would be involved later and later in the diagnostic process.

Representation of cause and effect in processes is starting to be addressed, for example by Palmer and Chung (2000) and Rengaswamy, Mylaraswamy, Arzen, and Venkatasubramanian (2001b). Automated linkage of cause and effect information from a process schematic with the results of data-driven analysis would be a fruitful direction for future research in order to test root cause hypotheses.

\section{Acknowledgements}

Nina Thornhill gratefully acknowledges the financial support of the Royal Academy of Engineering 
(Foresight Award). The project was also supported by the Natural Science and Engineering Research Council (Canada), Matrikon (Edmonton, Alberta) and the Alberta Science and Research Authority through the NSERC-Matrikon-ASRA Industrial Research Chair in Process Control, director Sirish Shah.

\section{References}

Åström, K. J. (1991). Assessment of achievable performance of simple feedback loops. International Journal of Adaptive Control and Signal Processing, 5, 3-19.

Bialkowski, W. L. (1992). Dreams vs. reality: A view from both sides of the gap. Proceedings of the Control Systems '92, Whistler, BC, Canada, pp. 283-329.

Choudhury, M. A. A. S., Shah, S. L., \& Thornhill, N. F. (2002). Detection and diagnosis of system nonlinearities using higher order statistics. Proceedings of the IFAC World Congress, Barcelona.

Ender, D. B. (1993). Process control performance: Not as good as you think. Control Engineering, 40(10), 180-190.

Forsman, K., \& Stattin, A. (1999). A new criterion for detecting oscillations in control loops. European Control Conference, Karlsruhe, Germany.

Hägglund, T. (1995). A control-loop performance monitor. Control Engineering Practice, 3, 1543-1551.

Horch, A. (1999). A simple method for detection of stiction in control valves. Control Engineering Practice, 7, 1221-1231.

Kantz, H., \& Schreiber, T. (1997). Nonlinear time series analysis. Cambridge, UK: Cambridge University Press.

Kayihan, A., \& Doyle, F. J. (2000). Friction compensation for a process control valve. Control Engineering Practice, 8, 799-812.

Martin, G. D., Turpin, L. E., \& Cline, R. P. (1991). Estimating control function benefits. Hydrocarbon Processing, 70(6), 68-73.

McMillan, G. K. (1995). Improve control valve response. Chemical Engineering Progress, 91(6), 76-84.

Miao, T., \& Seborg, D. E. (1999). Automatic detection of excessively oscillatory feedback control loops. IEEE Conference on Control Applications, Hawaii, pp. 359-364.

Palmer, C., \& Chung, P. W. H. (2000). Creating signed directed graph models for process plants. Industrial and Engineering Chemistry Research, 39, 2548-2558.
Paulonis, M. A., \& Cox, J. W. (2003). A practical approach for largescale controller performance assessment, diagnosis, and improvement. Journal of Process Control, 13, 155-168.

Piipponen, J. (1996). Controlling processes with nonideal valves: Tuning of loops and selection of valves. Proceedings of the Control Systems '96, Halifax, NS, Canada, pp. 179-186.

Qin, S. J. (1998). Control performance monitoring-a review and assessment. Computers and Chemical Engineering, 23, 173-186.

Rengaswamy, R., Hagglund, T., \& Venkatasubramanian, V. (2001a). A qualitative shape analysis formalism for monitoring control loop performance. Engineering and Applied Artificial Intelligence, 14, 23-33.

Rengaswamy, R., Mylaraswamy, D., Arzen, K. E., \& Venkatasubramanian, V. (2001b). A comparison of model-based and neural network-based diagnostic methods. Engineering Applications of Artificial Intelligence, 14, 805-818.

Ruel, M., \& Gerry, J. (1998). Quebec quandary solved by Fourier transform. Intech, 45(8), 53-55.

Schreiber, T., \& Schmitz, A. (2000). Surrogate time series. Physica D, 142, 346-382.

Shinskey, F. G. (2000). The three faces of control valves. Control Engineering, 47(7), 83.

Shunta, J. (1995). Achieving world class manufacturing through process control. Englewood Cliffs, NJ: Prentice-Hall.

Theiler, J., Eubank, S., Longtin, A., Galdrikian, B., \& Farmer, J. D. (1992). Testing for nonlinearity in time-series - the method of surrogate data. Physica D, 15, 77-94.

Thornhill, N. F., \& Hägglund, T. (1997). Detection and diagnosis of oscillation in control loops. Control Engineering Practice, 5, 1343-1354.

Thornhill, N. F., Huang, B., \& Zhang, H. (2003). Detection of multiple oscillations in control loops. Journal of Process Control, 13, 91-100.

Thornhill, N. F., Shah, S. L., \& Huang, B. (2001). Detection of distributed oscillations and root cause diagnosis. Proceedings of the CHEMFAS-4, Cheju, Korea, pp. 167-172.

Thornhill, N. F., Xia, C., Howell, J., Cox, J., \& Paulonis, M. (2002). Analysis of plant-wide disturbances through data-driven techniques and process understanding. Proceedings of the IFAC World Congress, Barcelona.

Whalen, B. R. (1983). Basic instrumentation (3rd ed.). Austin, TX: Petroleum Extension Service (PETEX).

Xia, C., \& Howell, J., (2003), Loop status monitoring and fault localisation. Journal of Process Control, in press, doi:10.1016/ S0959-1524(02)00123-3. 